This item was submitted to Loughborough's Research Repository by the author.

Items in Figshare are protected by copyright, with all rights reserved, unless otherwise indicated.

\title{
Acceptability and utilisation of patient-initiated follow-up for endometrial cancer amongst women from diverse ethnic and social backgrounds: A mixed methods study
}

\section{PLEASE CITE THE PUBLISHED VERSION}

https://doi.org/10.1111/ecc.12997

\section{PUBLISHER}

(c) Wiley

\section{VERSION}

AM (Accepted Manuscript)

\section{PUBLISHER STATEMENT}

This is the peer reviewed version of the following article: KUMARAKULASINGAM, P. ... et al, 2019. Acceptability and utilisation of patient-initiated follow-up for endometrial cancer amongst women from diverse ethnic and social backgrounds: A mixed methods study. European Journal of Cancer Care, 28 (2), e12997, which has been published in final form at https://doi.org/10.1111/ecc.12997. This article may be used for noncommercial purposes in accordance with Wiley Terms and Conditions for Use of Self-Archived Versions.

\section{LICENCE}

CC BY-NC-ND 4.0

\section{REPOSITORY RECORD}

Kumarakulasingam, Priyanga, Hilary McDermott, Nafisa Patel, Louise Boutler, Douglas G. Tincello, David Peel, and E.L. Moss. 2019. "Acceptability and Utilisation of Patient-initiated Follow-up for Endometrial Cancer Amongst Women from Diverse Ethnic and Social Backgrounds: A Mixed Methods Study". figshare. https://hdl.handle.net/2134/32406. 
1 ACCEPTABILITY AND UTILISATION OF PATIENT-INITIATED FOLLOW-UP

2 (PIFU) FOR ENDOMETRIAL CANCER AMONGST WOMEN FROM DIVERSE

3 ETHNIC AND SOCIAL BACKGROUNDS: A MIXED METHODS STUDY

4

$5 \quad{ }^{1} \mathbf{P}$ Kumarakulasingam BSc (medical student), ${ }^{2} \mathrm{H}$ McDermott PhD (lecturer in health

6 psychology), ${ }^{3} \mathrm{~N}$ Patel (clinical nurse specialist), ${ }^{3} \mathrm{~L}$ Boutler (clinical nurse specialist), ${ }^{4}$ DG Tincello MD (consultant gynaecologist), ${ }^{3}$ D Peel FRCR (consultant clinical oncologist), ${ }^{1,3}$ EL Moss PhD (consultant gynaecological oncologist)

$10{ }^{1}$ Department of Cancer Studies, University of Leicester, Leicester,

$11{ }^{2}$ School of Sport, Exercise and Health Sciences, Loughborough University,

$12{ }^{3}$ Department of Gynaecological Oncology, University Hospitals of Leicester

$13 \quad{ }^{4}$ Department of Health Sciences, University of Leicester, Leicester

Address for correspondence:

Dr Esther Moss PhD, ORCID 0000-0002-2650-0172, Department of Cancer Studies,

17 University of Leicester, Leicester Royal Infirmary, Leicester, LE2 7LX. Email: em321@1e.ac.uk. Tel: 00442584861

\section{FUNDING}

21 This study was funded by the University of Leicester and the University Hospitals of Leicester 22 Gynaecological Gynaecological Cancer Research fund.

\section{CONFLICT OF INTEREST STATEMENT}

None of the authors report conflict of interest. 
ABSTRACT

Introduction

30 A shift in focus towards risk stratification and survivorship in early stage endometrial cancer

31 (EC) has led to the replacement of hospital follow-up (HFU) with patient-initiated follow-up

32 (PIFU) schemes.

\section{Methods}

34 A mixed-methods study was undertaken prospectively to investigate utility and patient satisfaction with a newly introduced PIFU scheme.

\section{Results}

37228 women were enrolled onto PIFU in the first 18 months, median age 65 years (range 42-90 38 years). Twenty-four (10.5\%) women were non British-White ethnicity. Forty-five women contacted the Clinical Nurse Specialist (CNS) at least once (19.7\%), the primary reason being vaginal bleeding/discharge $(42 \%)$. Contact was greater in first six months on the scheme compared to the second six months, and women who made contact were significantly younger

42 than those who did not $(57$ years versus 65 years, $\mathrm{p}<0.001)$.

\section{Conclusions}

44 PIFU appears to be well received by the majority of women. Although many of the CNS contacts were due to physical symptoms, a number were for psychological support or reassurance. Younger women had greater CNS contact indicating that they may benefit from a greater level support. Patient feedback of the PIFU scheme was positive, with many women reporting that it enabled them to have more control over their own health.

KEYWORDS: Endometrial cancer; follow up; patient-initiated; quality of life; patient satisfaction 


\section{INTRODUCTION}

54 The incidence of endometrial cancer (EC) in the UK has increased by $65 \%$ over the past 40 years making it not only the most common gynaecological cancer but the fourth most common malignancy in women, accounting for $3 \%$ of all new cancer diagnoses (UK). The majority of cases are diagnosed with early stage disease, which carries a good long-term prognosis with 5year disease-specific survivals in excess of 95\% (Amant et al., 2005; UK). It is a disease typically affecting older women with the majority being over the age of 70 years at diagnosis and having co-morbidities, in particular obesity, diabetes and cardio-vascular disease (Parkin, Boyd, \& Walker, 2011).

62

Women diagnosed with an endometrial cancer have been traditionally followed-up through regular hospital review by a specialist gynaecological oncologist after completing their treatment (Colombo et al., 2011). Hospital follow-up (HFU) typically involves women being reviewed routinely by doctors and/or nurses who conduct a physical examination. The rationale for attending HFU is surveillance for cancer recurrence thus enabling early detection, prompt management and as a result a better long-term prognosis. The period of review has historically been for a minimum of five years since the majority of cancer recurrences occur within three years post treatment (Colombo et al., 2011). The role of routine follow-up in early stage, lowrisk endometrial cancer (EC) however has been questioned since it does not appear to have an impact of long-term survival (Gadducci, Cosio, Fanucchi, Cristofani, \& Genazzani, 2000; Owen \& Duncan, 1996; Yoshiba et al., 2016). In light of the rising incidence, low recurrence rate, population demographics and the lack clear clinical benefit of hospital follow-up in such cases, alternative follow-up schedules (Salani et al., 2011) and models have been developed, for example telephone follow-up (TFU) (Beaver et al., 2016) and patient-initiated follow-up (PIFU). 
79 PIFU, as the name suggests, is where patients are not routinely seen in hospital but instead have open access to Clinical Nurse Specialists (CNS) whom they can contact should they have concerns or if/when symptoms arise. The first UK national survey on the follow-up of gynaecological cancer patients reported that out of all the available follow-up schemes, PIFU was used instead of or as an adjunct to existing follow up models by $32 \%$ of the centres that responded (Leeson, Stuart, Sylvestre, Hall, \& Whitaker, 2013). Despite the wide spread use of PIFU there are very few reports in the literature examining patient views or satisfaction with such schemes.

87

A PIFU scheme was introduced in September 2014 at University Hospitals of Leicester (UHL) for early stage EC. Women who had received a diagnosis of early stage EC in the previous 5 years and were under HFU were offered transfer to the PIFU scheme. Women with a new diagnosis were started on PIFU immediately following completion of their treatment. PIFU was led by the CNSs who would have an end of treatment appointment with the patient where they would provide them with their contact details and written information on the signs/symptoms that should prompt a medical review. A telephone call by the CNS to the patient was scheduled for 6 and 12 months to ensure that the patient was still happy to continue on the PIFU scheme and had the contact details should symptoms arise. No other contact with

97 the medical team was organised.

98

In order to ensure patient satisfaction with the scheme a prospective study was conducted investigating patients' utilisation, personal opinions and attitudes towards the PIFU scheme. 


\section{METHODS}

104 A mixed methods study was undertaken following guidelines for qualitative research in order to ensure reliability and validity (BMJ, 2016). Mixed methods studies are increasingly used

106 within health research to increase our understanding of health problems (Plano Clark \&

107 Creswell, 2010). This study utilized a sequential explanatory approach (Terrell, 2012) whereby

108 the results from the distributed questionnaires informed the development of the semi-structured interview schedule. In addition, qualitative data added depth and understanding to the

110 questionnaire findings.

112 The introduction of the PIFU scheme at UHL was supported by the East Midlands

113 Gynaecological Oncology Clinical Advisory Group (ECAG) and the UHL Cancer Board. The 114 prospective audit of patient utility and satisfaction of the scheme was approved by the

115 University Hospitals of Leicester audit team. Ethical approval was granted for the patient 116 interviews (15/WM/0239). Women with a diagnosis of early stage low-risk EC and early stage

117 intermediate risk EC who had completed adjuvant treatment, were enrolled onto the PIFU 118 scheme in its first 18 months (September 2014 to March 2016) and were included in the study.

\section{Scheme utilisation}

121 Information was collated on the demographics of the patients, including age at diagnosis and

122 ethnicity. The contemporaneous call log and individual patient records kept by the CNSs were

123 reviewed and classified by primary reported reason for telephone contact: physical; 124 psychological; practical. The data were analysed using GraphPad Prism (GraphPad Software, 125 Inc, La Jolla, USA). 
129 Quality of Life (EORTC QLQ-C30) and patient satisfaction questionnaires (Appendix 1) were

130 posted to the women on the scheme at 6 and 12 months following enrolment. The QLQ-C30

131 results were analysed using the EORTC QLQ-C30 scoring manual (Fayers \& Bjordal, 2001)

132 in order to give a score for Functioning, Symptoms and Global Health Status and were

133 compared against the references values (Scott \& Aaronson, 2008).

\section{Semi-Structured interviews}

136 Women enrolled on the PIFU scheme were invited to attend a face-to-face semi-structured

137 interview with two female members of the research team (PK and EM) to discuss their views and opinions on PIFU. Both PK and EM are trained in interview techniques. One of the interviewers was a member of the clinical gynaecological oncology team at the University Hospitals of Leicester. In order to achieve a wide sampling frame women were grouped in to

141 British White (BW) (Group a) and non British White (non BW) (Group b) and then subdivided 142 into women who transferred onto the PIFU scheme from HFU (Group A) and those who were 143 started directly onto the PIFU scheme following their diagnosis (Group B). Women were 144 selected randomly from each of the four groups ( $\mathrm{Aa}, \mathrm{Ab}, \mathrm{Ba}, \mathrm{Bb}$ ) by hospital unit number and a written invitation was posted to their home address. Invitations were sent out and interviews

146 were conducted in an outpatients clinic room, until data saturation was achieved. An interview

147 guide was used asking questions on their treatment journey, experiences and views on different

148 follow up schemes and views of the future. Interviews were recorded with the knowledge and consent of the participants and subsequently transcribed verbatim. Due to the interviews being fully transcribed, field notes were not undertaken by either interviewer. 
152 The interview data were analysed using the six-stage process of Thematic Analysis described

153 by Braun \& Clarke (2006) and managed using Nvivo software. Initially, the analysis was

154 deductive but as the analysis progressed it became more inductive as new themes were

155 identified in the data. The data transcripts were read and re-read to facilitate familiarisation

156 with the data, and then coded line-by-line by the researchers (PK and HM). Repeated reading

157 of the data allowed codes to be developed, which were subsequently grouped into themes. As

158 the analysis progressed key themes were selected and further sub-themes were subsequently

159 developed. A thematic map incorporating the identified codes was created in order to identify

160 trends and associations. To ensure the trustworthiness of the coding the two researchers (PK

161 and HM) independently coded the data and during discussions similar codes and interpretations

162 were found to have been applied to the cross checked sections of data. All experiences reported

163 by participants were included in the analysis and represented in the manuscript. Triangulation

164 was used at the interpretation stage of the study in order to consider the findings from the

165 quantitative and qualitative aspects and determine their agreement or dissonance (Erzberger \&

166 Prein, 1997).

167

168

169 RESULTS

170 Over the 18-month study period 228 women with a diagnosis of an early stage EC were 171 enrolled onto the PIFU scheme. The median age was 65 (range 42 - 90 years). The majority of 172 women, 204 (89.5\%), were BW and 24 (10.5\%) were non BW (22 British South Asian (BSA) and 2 African/AfroCaribbean (AA)). One hundred and three women were diagnosed pre-

174 September 2014 and were transferred from routine hospital follow-up (HFU) onto the PIFU 175 scheme. There were 125 women diagnosed post-September 2014 that were directly transferred 176 onto the PIFU scheme. The median time on the PIFU scheme was 14 months (95\% CI 12.9- 
17714.3 months). The total number of women in follow-up prior to September 2014 or who had completed adjuvant therapy was not known and therefore it was not possible to calculate the proportion of patients who moved to PIFU. Women in these groups were given the choice of PIFU rather than HFU by their supervising clinicians. Of the women diagnosed post-September 2014 with early stage low-risk EC less than 5\% were deemed not suitable for recruitment to PIFU, primarily due to mental health issues.

\section{Patient-Initiated Contact}

Forty-five women (19.7\%) contacted the Clinical Nurse Specialists (CNS) at least once. Significantly more contact was made in the first six months of being entered onto the scheme as compared to the second six months $(37 / 45$ versus $8 / 45$, Chi squared $p<0.001)$. Women who were transferred onto PIFU directly also were more likely to make contact compared to women who had been under routine HFU and then transferred (30/125 women versus 15/103 women, Chi square $\mathrm{p}=0.075$ ) and were significantly younger than women who did not contact (median age 57 years compared to 65 years, Mann Whitney $\mathrm{p}<0.001)$. Although women of non BW ethnicity were more likely to contact $(6 / 24,33.3 \%)$ compared to BW women $(39 / 204,19.1 \%)$ the difference was not statistically significant. Four women were transferred from PIFU back to HFU due to patient anxiety and multiple symptomatology.

The primary reason for contacting the CNS were mainly due to physical symptoms (62\%), such as vaginal bleeding/discharge or abdominal pain, however, $20 \%$ of the calls were for psychological support or reassurance. Another 2 calls were for practical assistance, for example help claiming benefits. 
In total, 153 6-month and 109 12-month Quality of Life (QLQ) and Patient Satisfaction (PSQ) (FSS) between the six-month and 12-month QLQs (Figure 1). For all of the functioning subscales, the scores from both 6 and 12 months were higher than the references values, indicating that the women on the PIFU scheme had a higher level of functioning than the reference populations 'all cancer patients: female' and 'all cancer patients: stage 1-II'. The scores were also lower than the reference values for the symptoms scale score (SSS) (Figure 2) and for the global health status score (GHSS) (Figure 3). There was no difference in the

211 GHSS score between the 6- and 12-month QLQs, Mann Whitney $p=0.564$.

\section{Patient Satisfaction}

214 Patient feedback regarding the PIFU scheme was positive, with $62 \%$ of women in the 6-month PSQ reported they found it useful (very much/quite a bit) and only one patient $(0.6 \%)$ reporting

216 that they did not find the scheme useful at all. Dissatisfaction increased slightly at the 12-month

217 PSQ with 4 women (3.6\%) giving the response 'not at all' to the question 'Overall how reassured are you by PIFU?' but this contrasted with $63 \%$ of women who responded very much/quite a bit. In the PSQs 25 women (six-month PSQ) and 12 women (12-month PSQ) reported contacting the CNS with the most common reason being that they were worried about a symptom they had developed. The next most commonly reported reason was that they wanted to speak about worries and feelings that they were having.

\section{Patient Attitudes}

225 Of the 51 women contacted, 21 women agreed to (13 BW and 8 non BW) participate in a semi226 structured interview. Non BW women were offered translators, which two accepted and two 
women brought a family member with them to translate. Interview times ranged from 20 to 58 minutes.

230 PIFU was reported as being the preferred follow-up model by nine women. Four women 231 preferred HFU and five suggested a combination of HFU initially following treatment with 232 transfer to PIFU after a short period of time, for example 12 months, would be better. Women that preferred the PIFU scheme commented on the importance of being able to carry on with their lives and being able to have more control over their own health whilst having the reassurance of direct access to the CNS if needed. For example, one 53-year-old non BW

236 female stated; "It stops me having to worry about 'I've got an appointment here to come and see this person'. I'm looking out for my own symptoms and know that if I ring up the secretary or the clinic and say 'I have this issue, can I come and see somebody?' I can come in. I don't have to go via the GP is what I'm saying." The main reason given for preferring hospital follow-up (HFU) was the reassurance of seeing somebody face-to-face as it was thought to be more personal. A 51-year-old BW female said; "I think I would have preferred to come back and seen, physically seen someone... I think it's more just reassurance to meet somebody face to face about it. It's a bit more personal." No participants reported that they would have preferred to be seen by their general practitioner (GP) for follow-up.

246 The majority of women on the PIFU scheme understood how to use the scheme. The overall

247 feeling reported by participants was that it was easier and quicker to contact the CNS and they reported that they had confidence that the CNS would instigate any necessary investigations or appointments, rather than being seen in primary care for symptoms. The majority of the non BW women interviewed were of BSA ethnicity and they felt particularly reassured that they could communicate with a Gujarati/Hindi speaking CNS. A 59-year old non BW female said; 
'Yes. It's very helpful because it's in our language, whatever the problem is I can communicate it very well and I'm happy".

The main reason participants reported for not utilising the scheme was the fear of wasting the CNS' time. For example, a 63 year-old BW female stated; "It's too easy to think 'I won't bother' because it, I don't know, you feel like you're bothering somebody." Other reported reasons included either worrying about or ignoring symptoms participants thought might have been related to their endometrial cancer diagnosis. For example, a 53-year old non BW female said; "The only barrier that I think would stop them ringing in is if they worried that it has come back. Because you've got to get your mind around that one first before you go and ring”.

'Wasting the doctor's time' and the 'unnecessary journey' to a HFU appointment if women were asymptomatic were commonly reported barriers to attending HFU. A 63 year-old BW female said; 'It becomes a bit of a pain coming in every 4 months, every 6 months, when actually there's not anything wrong with you and it's a waste of your time, bus money, petrol money, whatever the consultant's time, when there's actually nothing wrong with you.'

DISCUSSION

Risk stratification with regard to patient follow-up is not a new concept in oncology. PIFU schemes have been developed for low-risk disease for several reasons, both patient and economic, across a range of specialties and diseases (Taneja, Su'a, \& Hill, 2014). However, ensuring patient safety and satisfaction has to be the central goal when evaluating such schemes. In this mixed methods study we have used both quantitative and qualitative methods to ensure women are utilising PIFU and are accessing the CNSs when needed. The results of the two investigative methods have given complimentary information, with the quantitative 
aspect determining the frequency/indication for contact and the interviews exploring women's

278 rationale for their behavior. Leeson et al., (Leeson et al., 2013) identified that there are a wide range of follow-up models currently in use in the UK for women who have been diagnosed with a gynaecological malignancy. Many centres reported using telephone (25\%) or patientinitiated (32\%) follow up schemes, in contrast to ESMO guidance (Colombo et al., 2011), which supports a policy of regular follow up for all patients for 5 years.

We have demonstrated that early stage EC patients experience a good quality of life post treatment, noticeably higher than the reference populations 'all cancer patients: female' and 'all cancer patients: stage I-II' used for the EORTC-QLQ30. A strong theme that emerged in the interviews was that women understood that their risk of recurrence was low and did not want to keep being reminded of their diagnosis by attending HFU. This may be interpreted as a self-management coping mechanism whereby the participants tried to leave their cancer experiences behind them in an attempt to adopt some form of normality. Indeed, reminders of cancer have been found to induce fear and increased insecurity of cancer recurrence in breast cancer survivors (Drageset, Lindstrom, \& Underlid, 2016).

The fear of cancer recurrence can lead to cancer survivors employing specific self-management strategies to forget their cancer diagnosis. Howard et al., (Howard et al., 2016) identified that adult childhood cancer survivors' employed strategies such as avoiding contact with medical personnel and not attending hospital/clinic appointments in an attempt to forget their previous diagnosis. In a further study by Zelman et al., (Zelman et al., 2004) participants reported that being able to forget about their cancer diagnosis, even for a short while, was an important determinant of a 'desirable day'. Returning to normal life has been identified as a highly motivating factor for recovery (Howard et al., 2016) and an important factor in coping with a 
cancer diagnosis (Drageset, Lindstrom, \& Underlid, 2010). PIFU removes the need for patients

to attend hospital appointments and it may be argued that this can facilitate their 'return to

304

305

306

307

308

309

310

311 We have shown that PIFU for early stage EC is well received by our population. A recent normal'. The good long-term prognosis of early stage EC and PIFU can help to change people's perception that cancer is not necessarily a fatal disease but that they can have a life beyond their diagnosis. The change in focus toward patient survivorship is increasingly necessary and the importance of developing supported self-management pathways, helping patients to manage their own health and wellbeing can not only address unmet needs but can reduce the demand on support services.

randomised trial evaluating the effectiveness of nurse-led telephone follow-up (NTFU), compared to HFU, also reported high levels of patient satisfaction, with no detrimental physical or psychological effects (Beaver et al., 2016). Our scheme was not classified as a NTFU since the 6-monthly calls were not structured and did not ask questions on symptoms or wellbeing, merely ensured that the patient understood the purpose of the PIFU scheme and was happy to remain on it. We did identify that younger women and women from a non BW ethnicity were more likely to initiate contact, suggesting that they were in greater need of support from the CNS. A reason for this finding could be that psychological unmet needs have been shown to be significantly associated with age at diagnosis (Boyes, Girgis, D'Este, \& Zucca, 2012). BSA cancer patients are reported to have twice the rate of self-reported depressive symptoms and five times the incidence of severe depression as compared to BW cancer patients (Lord et al., 2013). Lord et al., (Lord et al., 2013) also reported that BSA patients used more maladaptive coping strategies, such as helplessness/hopelessness and fatalism, and experienced a heavier physical symptom burden as compared to BW patients. In our study the BSA women reported that they were very supportive of PIFU preferring not to attend the hospital, since this was felt 
to be a sign of ongoing active disease rather than surveillance for disease that had been treated.

328 It is essential when designing a PIFU scheme to ensure that the women who are enrolled have the capacity and insight to contact the CNS should an issue arise. Women who are non-English

330 speakers are at particular risk of being disenfranchised and education sessions with their

331 family/carers and the availability of interpreting services can enable women to participate in

332 PIFU. Knowledge of the local community and its demographics can be helpful in planning services, for example Leicester has a high BSA population who are predominantly Gujarati speaking. An advantage of the Leicester PIFU scheme therefore is that one of the CNSs is a fluent Gujarati/Hindi speaker thereby enabling non-English speakers to access the scheme, another positive factor reported by the non BW women who were interviewed.

\section{CONCLUSION}

PIFU for early stage EC appears to be well received by the majority of women. Although many of the CNS contacts were related to physical symptoms, many were for psychological support or reassurance. Younger women had greater CNS contact indicating that they may benefit from a greater level of CNS support. Women from a non WB ethnicity are also supportive of PIFU, although consideration should be made to ensure non-fluent English speakers are able to access the scheme. 


\section{REFERENCES}

Amant, F., Moerman, P., Neven, P., Timmerman, D., Van Limbergen, E., \& Vergote, I. (2005). Endometrial cancer. Lancet, 366(9484), 491-505. doi:10.1016/S01406736(05)67063-8

Beaver, K., Williamson, S., Sutton, C., Hollingworth, W., Gardner, A., Allton, B., . . Martin-Hirsch, P. (2016). Comparing hospital and telephone follow-up for patients treated for stage-I endometrial cancer (ENDCAT trial): a randomised, multicentre, non-inferiority trial. BJOG. doi:10.1111/1471-0528.14000

BMJ Editor's checklists 2016 [cited 20161 April]. Available from: http://www.bmj.com/about-bmj/resources-authors/article-types/research/editorschecklists.

Boyes, A. W., Girgis, A., D'Este, C., \& Zucca, A. C. (2012). Prevalence and correlates of cancer survivors' supportive care needs 6 months after diagnosis: a population-based cross-sectional study. BMC Cancer, 12, 150. doi:10.1186/1471-2407-12-150

Colombo, N., Preti, E., Landoni, F., Carinelli, S., Colombo, A., Marini, C., . . . Group, E. G. W. (2011). Endometrial cancer: ESMO Clinical Practice Guidelines for diagnosis, treatment and follow-up. Ann Oncol, 22 Suppl 6, vi35-39. doi:10.1093/annonc/mdr374

Drageset, S., Lindstrom, T. C., \& Underlid, K. (2010). Coping with breast cancer: between diagnosis and surgery. $J$ Adv Nurs, 66(1), 149-158. doi:10.1111/j.13652648.2009.05210.x

Drageset, S., Lindstrom, T. C., \& Underlid, K. (2016). "I just have to move on": Women's coping experiences and reflections following their first year after primary breast cancer surgery. Eur J Oncol Nurs, 21, 205-211. doi:10.1016/j.ejon.2015.10.005

Erzberger, C., \& Prein, G. (1997). Triangulation: Validity and empirically-based hypothesis construction. Quality \& Quantity, 31(2), 141-154.

Fayers, P., \& Bjordal, K. (2001). EORTC QLQ-C30 scoring manual (3rd ed.). Brussels: EOTOC Publications.

Gadducci, A., Cosio, S., Fanucchi, A., Cristofani, R., \& Genazzani, A. R. (2000). An intensive follow-up does not change survival of patients with clinical stage I endometrial cancer. Anticancer Res, 20(3B), 1977-1984.

Howard, A. F., Goddard, K., Tan de Bibiana, J., Pritchard, S., Olson, R., \& Kazanjian, A. (2016). Adult childhood cancer survivors' narratives of managing their health: the unexpected and the unresolved. J Cancer Surviv, 10(4), 711-725. doi:10.1007/s11764-016-0517-8

Leeson, S., Stuart, N., Sylvestre, Y., Hall, L., \& Whitaker, R. (2013). Gynaecological cancer follow-up: national survey of current practice in the UK. BMJ Open, 3(7). doi:10.1136/bmjopen-2013-002859

Lord, K., Ibrahim, K., Kumar, S., Mitchell, A. J., Rudd, N., \& Symonds, R. P. (2013). Are depressive symptoms more common among British South Asian patients compared with British White patients with cancer? A cross-sectional survey. BMJ open, 3(6), e002650.

Owen, P., \& Duncan, I. D. (1996). Is there any value in the long term follow up of women treated for endometrial cancer? Br J Obstet Gynaecol, 103(7), 710-713.

Parkin, D. M., Boyd, L., \& Walker, L. C. (2011). 16. The fraction of cancer attributable to lifestyle and environmental factors in the UK in 2010. Br J Cancer, 105 Suppl 2, S7781. doi:10.1038/bjc.2011.489 
Plano Clark, V., \& Creswell, J. (2010). Understanding research: a consumer's guide. Upper Saddle River, NJ: Prentice Hall - Merrill.

Salani, R., Backes, F. J., Fung, M. F., Holschneider, C. H., Parker, L. P., Bristow, R. E., \& Goff, B. A. (2011). Posttreatment surveillance and diagnosis of recurrence in women with gynecologic malignancies: Society of Gynecologic Oncologists recommendations. Am J Obstet Gynecol, 204(6), 466-478. doi:10.1016/j.ajog.2011.03.008

Scott, N., \& Aaronson, N. (2008). EORTC QLQ-C30 Reference Values. Brussels: EORTC Publications.

Taneja, A., Su'a, B., \& Hill, A. G. (2014). Efficacy of patient-initiated follow-up clinics in secondary care: a systematic review. Intern Med J, 44(12a), 1156-1160. doi:10.1111/imj.12533

UK, C. R. Survival statistic for womb cancer | Cancer Research UK. Cancerresearchuk.org. 2016 [cited 12 July 2016]. Available from: http://www.cancerresearchuk.org/aboutcancer/type/womb-cancer/treatment/statistics-and-outlook-for-womb-cancer\#prog.

UK, C. R. Uterine cancer incidence statistics 2016 [cited 201613 May]. Available from: http://www.cancerresearchuk.org/health-professional/cancer-statistics/statistics-bycancer-type/uterine-cancer/incidence\#heading-Two.

Yoshiba, T., Takei, Y., Machida, S., Taneichi, A., Sato, N., Takahashi, S., . . . Fujiwara, H. (2016). Prognosis of endometrial cancer patients with and without symptoms at recurrence. J Obstet Gynaecol Res. doi:10.1111/jog.13112

Zelman, D. C., Smith, M. Y., Hoffman, D., Edwards, L., Reed, P., Levine, E., . . Dukes, E. (2004). Acceptable, manageable, and tolerable days: patient daily goals for medication management of persistent pain. J Pain Symptom Manage, 28(5), 474-487. doi:10.1016/j.jpainsymman.2004.02.022

(1)

(1)

(1)


486

487

488

489

490

491

492

493

494

Figure 1.

EORTC Functioning Scale Scores (FSS).

\section{A) Physical functioning.} 6 months $\mathrm{n}=138,12$ months $\mathrm{n}=104$. Mann Whitney $\mathrm{p}=0.938$.

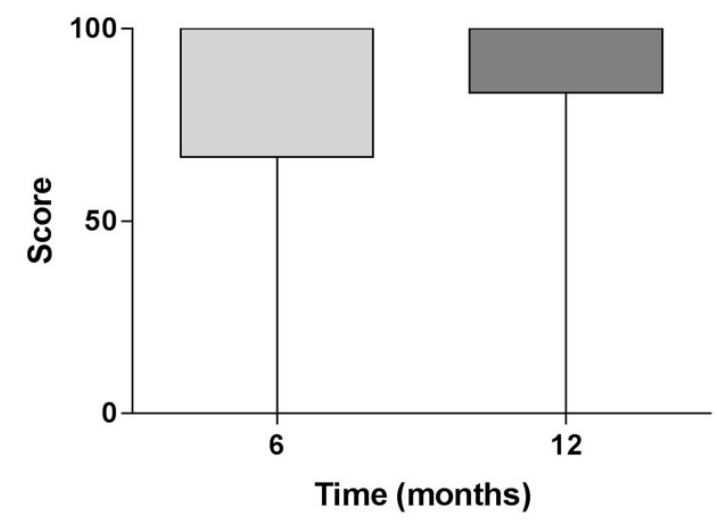

B) Emotional functioning.

6 months $n=143,12$ months $n=101$. Mann Whitney $\mathrm{p}=0.813$.

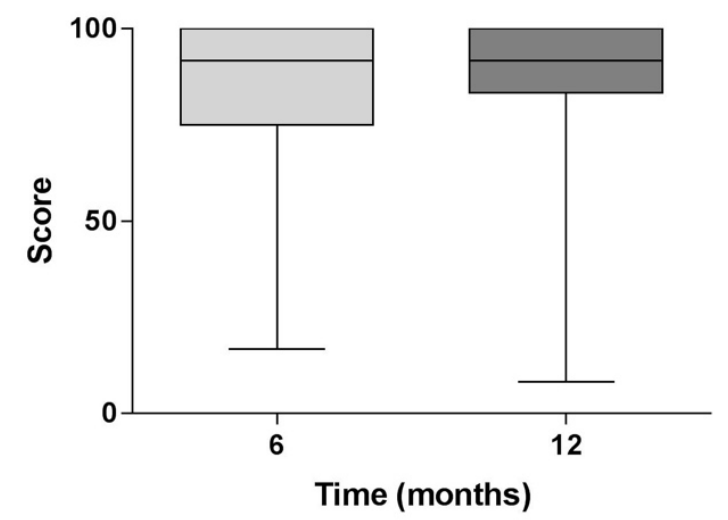

\section{C) Role functioning}

6 months $n=147,12$ months $n=104$. Mann Whitney $p=0.563$

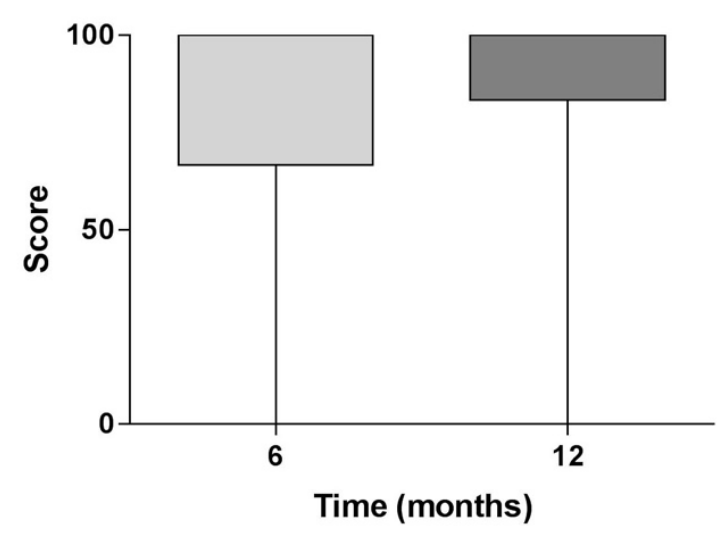


Figure 2. Symptoms scale score (SSS).

A) Pain

6 months $n=145,12$ months $n=105$. Mann Whitney $p=0.429$

500

501

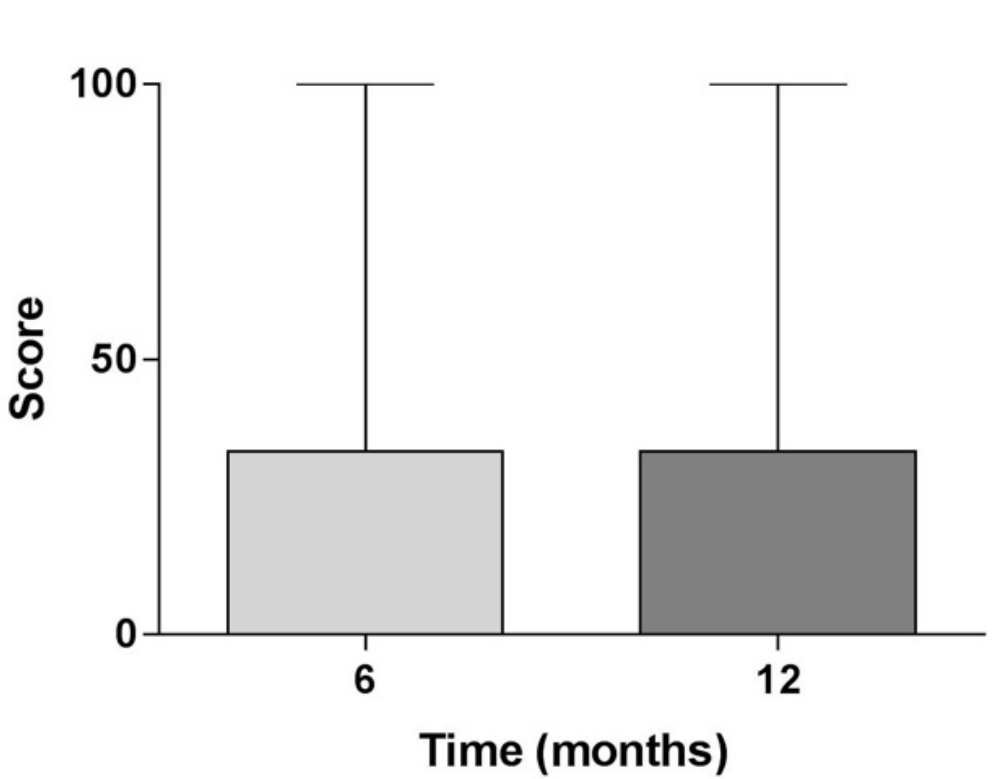

520

\section{B) Fatigue}

6 months $n=146,12$ months $n=104$. Mann Whitney $p=0.250$

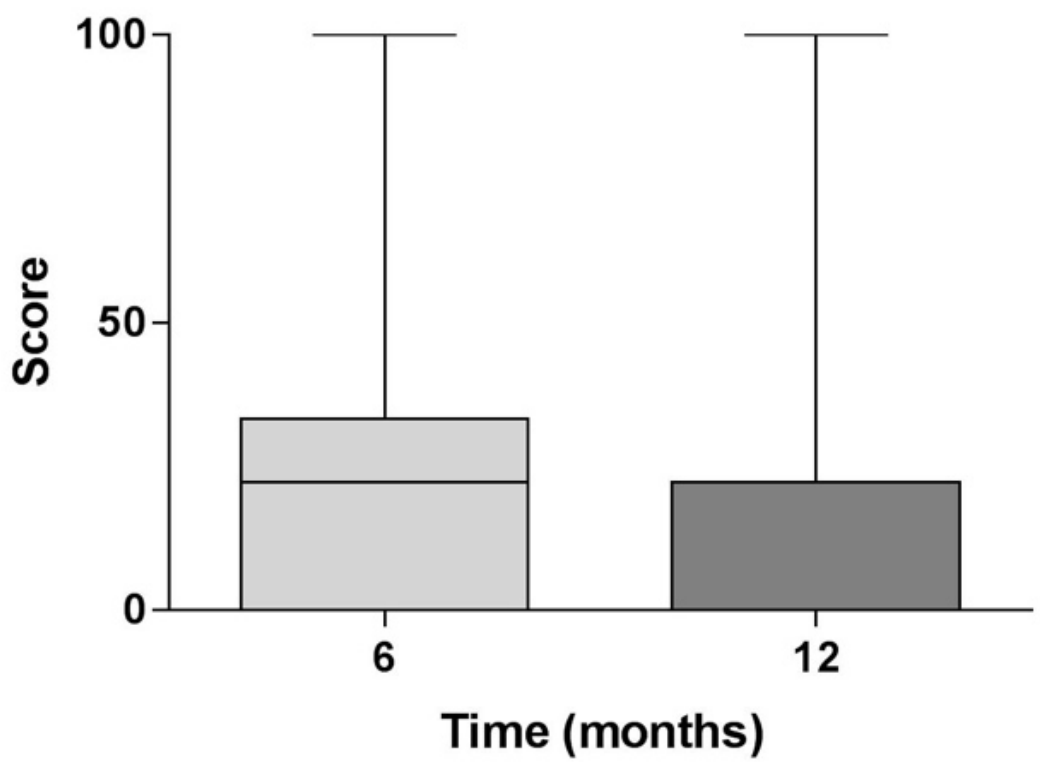


539 Figure 3. EORTC Global Health Status Scores (GHSS). Range from 0-100 with a high score 540 indicating a high quality of life. 6 months $n=146,12$ months $n=106$. Mann Whitney $p=0.564$. 541

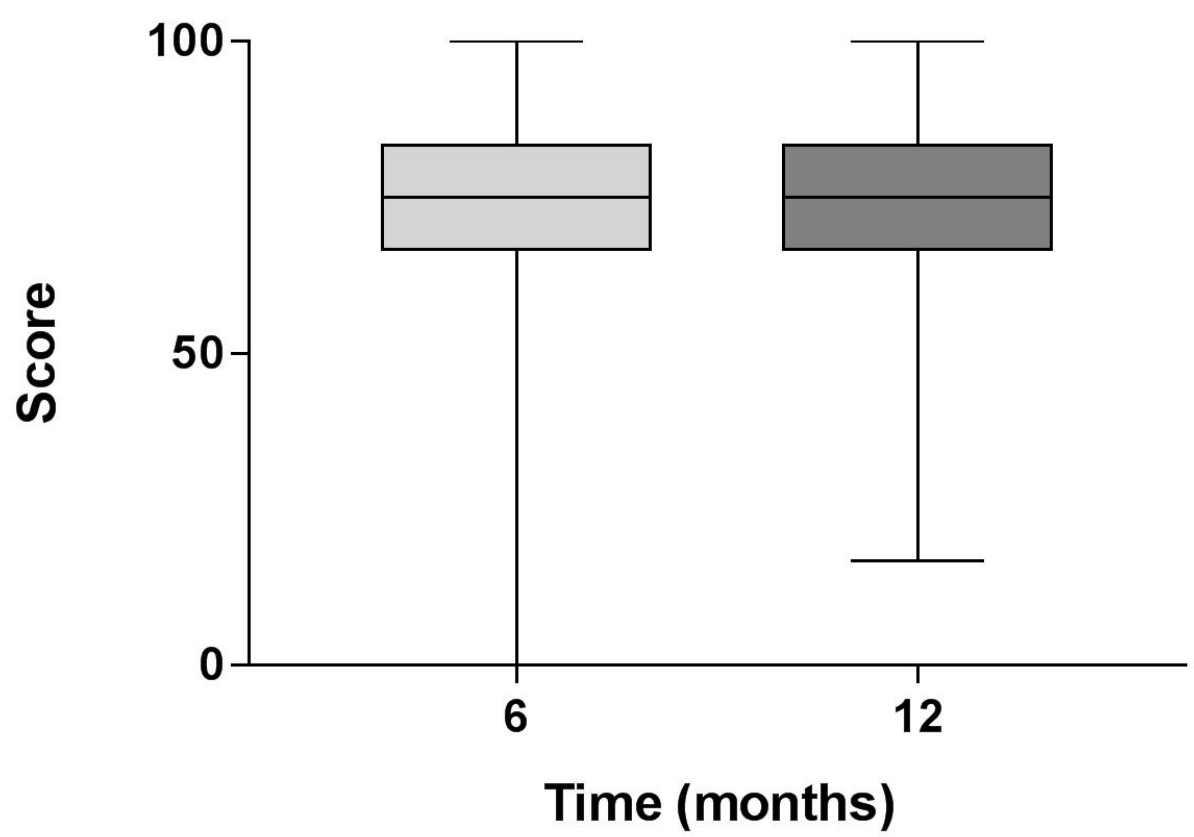

